\title{
The Dynamic Influence of Linker Histone Saturation within the Poly-Nucleosome Array
}

\author{
Dustin C. Woods ${ }^{1}$, Francisco Rodríguez-Ropero ${ }^{2}$, and Jeff Wereszczynski ${ }^{2}{ }^{*}$ \\ ${ }^{1}$ Department of Chemistry and the Center for Molecular Study of Condensed Soft Matter, \\ Illinois Institute of Technology, Chicago, IL 60616 \\ ${ }^{2}$ Department of Physics and the Center for Molecular Study of Condensed Soft Matter, \\ Illinois Institute of Technology, Chicago, IL 60616 \\ ${ }^{*}$ To whom correspondence should be addressed: jwereszc@iit.edu
}

September 21, 2020 


\begin{abstract}
Linker histones bind to nucleosomes and modify chromatin structure and dynamics as a means of epigenetic regulation. Biophysical studies have shown that chromatin fibers can adopt a plethora of conformations with varying levels of compaction. Linker histone condensation, and its specific binding disposition, has been associated with directly tuning this ensemble of states. However, the atomistic dynamics and quantification of this mechanism remains poorly understood. Here, we present molecular dynamics simulations of octa-nucleosome arrays, based on a cryo-EM structure of the 30-nm chromatin fiber, with and without the globular domains of the H1 linker histone to determine how they influence fiber structures and dynamics. Results show that when bound, linker histones inhibit DNA flexibility and stabilize repeating tetra-nucleosomal units, giving rise to increased chromatin compaction. Furthermore, upon the removal of $\mathrm{H} 1$, there is a significant destabilization of this compact structure as the fiber adopts less strained and untwisted states. Interestingly, linker DNA sampling in the octa-nucleosome is exaggerated compared to its mono-nucleosome counterparts, suggesting that chromatin architecture plays a significant role in DNA strain even in the absence of linker histones. Moreover, H1-bound states are shown to have increased stiffness within tetra-nucleosomes, but not between them. This increased stiffness leads to stronger long-range correlations within the fiber, which may result in the propagation of epigenetic signals over longer spatial ranges. These simulations highlight the effects of linker histone binding on the internal dynamics and global structure of poly-nucleosome arrays, while providing physical insight into a mechanism of chromatin compaction.
\end{abstract}

\title{
Significance
}

Linker histones dynamically bind to DNA in chromatin fibers and serve as epigentic regulators. However, the extent to which they influence the gamut of chromatin architecture is still not well understood. Using molecular dynamics simulations, we studied compact octa-nucleosome arrays with and without the H1 linker histone to better understand the mechanisms dictating the structure of the chromatin fiber. Inclusion of $\mathrm{H} 1$ results in stabilization of the compact chromatin structure, while its removal results in a major conformational change towards an untwisted ladder-like state. The increased rigidity and correlations within the H1-bound array suggests that H1-saturated chromatin fibers are better suited to transferring long-range epigentic information. 


\section{Introduction}

Serving as the primary storage vessel of genomic information within eukaryotic organisms, chromosomes consist predominantly of organized, long condensed fibers of DNA and structural proteins.1 Known as chromatin, these fibers are made of compacted repeating arrays of distinct DNA-protein complexes called nucleosomes. ${ }^{14}$ Nucleosomes consist of $\sim 147$ bp of DNA wrapped around an octamer core of organized histone proteins. ${ }^{[5]}$ Within the array, nucleosomes are inter-spaced between varying lengths of linker DNA, which is often quantified by their nucleosome-repeat-length (NRL) ${ }^{6}$ Computational modeling and topological studies have shown that the NRL regularity can directly affect chromatin compaction via variations in local fiber stiffness. ${ }^{718}$ Furthermore, this value can depend on interactions with a variety of cosolute compounds, nucleosome remodeling factors, ${ }^{6}$ or DNA sequence ${ }^{9}$ usually related to a level of charge neutralization and/or structural accommodation. Some examples include cosolute cations (i.e. $\mathrm{Mg}^{2+}$, nuclear polyamines, etc.), basic amino acids found on the terminal tail domains of core histones, proteins found outside of the nucleosome core, and H1 linker histones. 10,15

Structural studies have shown chromatin fibers adopt multiple states, including solenoid ${ }^{[6] 17}$ or zigzag $\sqrt{18} \sqrt[23]{23}$ like-conformations, with evidence of both forms being present within the same fiber. ${ }^{24}$ At high ionic strength, nucleosome arrays compact to create fibers with a diameter of about 30-nm in a closed zigzag conformation, $22 \sqrt[25 \mid 29]{29}$ similar to what is shown in Figure 1. However, despite the fact that canonical chromatin does form chains with regular and irregular zigzag structures, there is a particular absence of 30-nm fibers from eukaryotic nuclei, $, 30,37$ except within terminally differentiated cells. 38,41

To date, chromatin structural and mechanistic studies have largely focused on structural regulation at the single-nucleosomal level, ${ }_{42}^{42}$ including such phenomena as nucleosome opening, 43,45 the influence of extra-nucleosomal proteins, $\underline{46} \underline{48}$ and histone variants. $\frac{49}{9}$ More recently, studies involving poly-nucleosomal arrays and models of higher order structures have begun to show that chromatin exists in a dynamic equilibrium of states, $2433[37|50| 52]$ suggesting that it exhibits large-scale, concerted dynamics orchestrated by motifs such as remodeling factors and histone variants. Moreover, contemporary coarse-grained modeling of poly-nucleosome arrays with $\mathrm{H} 1$ have further emphasized the diversity of chromatin dynamics highlighting structures with irregular NRLs,,$[50]$ varied cation concentrations, 2451 and higher order structures. ${ }^{[3752}$ However, there is a severe lack of atomistic resolution studies of poly-nucleosome arrays 53154 which may provide residue-specific information otherwise lost by coarse-grained models. Interacting with both core and linker DNA, 10 the linker histone (H1) plays a crucial role in the condensation of nucleosome chains into higher order architecture, ${ }^{48|55| 57}$ like the zig-zag structure, ${ }^{37}$ along with other cellular functions ${ }^{\sqrt{57}}$ such as gene ex- 


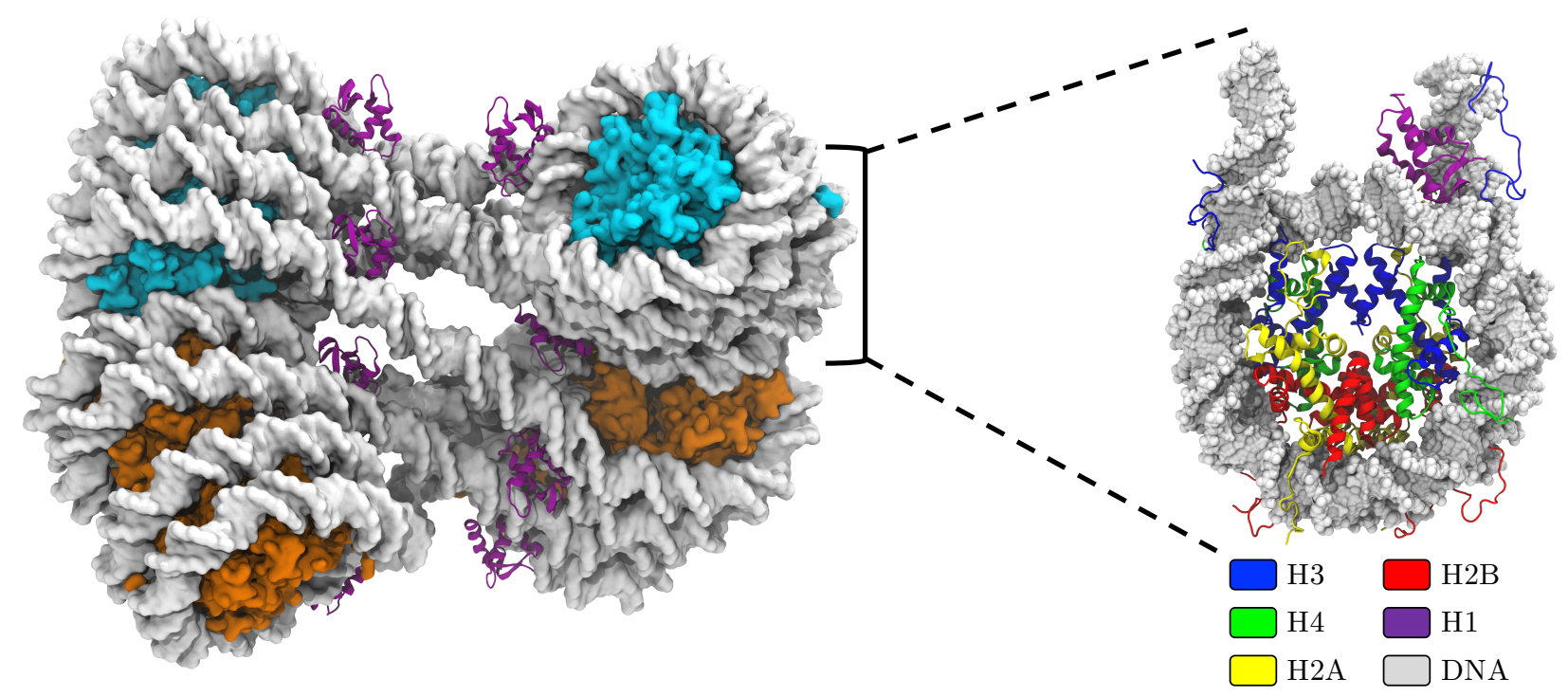

Figure 1: Shown on the left is the octa-nucleosome array constructed using the cryo-EM map of the $30 \mathrm{~nm}$ chromatin fiber. 22 Each nucleosome is paired with a linker histone (purple) bound asymmetrically off the dyad axis. On the right is an example of a mono-nucleosome unit from the array with each individual histone shown. The core histones in the poly-nucleosome (left) are colored cyan and orange to distinguish between the upper and lower tetra-nucleosome sub-units.

pression, ${ }^{58[59}$ heterochromatin genetic activity, $\frac{60}{60}$ and cell differentiation, ${ }^{61 \mid 62}$ among many others. $\frac{63,65}{65}$ They are found roughly every $200 \pm 40$ base pairs,,$[66$ but may be spaced more intermittently to regulate DNA accessibility for transcription factors. Additionally, linker histones predominantly interact electrostatically with the backbone phosphates of DNA using positively charged residues, $\underline{67-69}$ which stabilizes nucleosome arrays hindering linker DNA accessibility and competing with core histone tails for binding space. $26 \sqrt{48 / 70} 73$ However, this effect has been shown to be completely abrogated upon the addition of nucleosome-free regions within H1-saturated arrays. 74

In a previous study, we used all-atom molecular dynamics (MD) simulations to demonstrate that the linker histone binding mode on nucleosomes can have substantial effects on linker DNA dynamics. $\frac{75}{75}$ Furthermore, we postulated that its presence would have cascading effects on higher order chromatin structures. Indeed, this is highlighted in many of the previously mentioned studies, but none of which provide a mechanism detailing the atomistic dynamics of the chromatin fiber in and out of the presence of H1. To extend these ideas to the chromatin fiber, we examined these dynamics via all-atom MD simulations of an octa-nucleosome array with and without the $D$. melanogaster generic globular domain of H1 bound asymmetrically off the dyad. Results suggest that linker histones provide stabilization to the fiber structure at multiple levels. Helical parameters, inspired by similar DNA base-pairing metrics, ${ }^{[76}$ quantified a major conformational 
shift from a twisted condensed state to an untwisted ladder-like state. Stiffness parameters of these metrics show H1 binding increases torsional stress within tetra-nucleosome sub-units. Furthermore, while an angular analysis of linker DNA motions shows that linker histones limit sampling, it also highlights the stark contrast in mono- versus poly-nucleosome dynamics, especially among in-plane DNA motions. Moreover, generalized correlation analyses shows that linker histone saturation strengthens long-range correlations throughout each system, which can lead to further transfer of epigenetic information across the fiber. This linker histone saturation provides stabilization to the highly strained linker DNA resulting in a highly compact system that is unfavorable for transcription factor binding. Complete abrogation of these extra-nucleosomal proteins allows the fiber to untwist and thus alleviating the aforementioned linker DNA strain. 


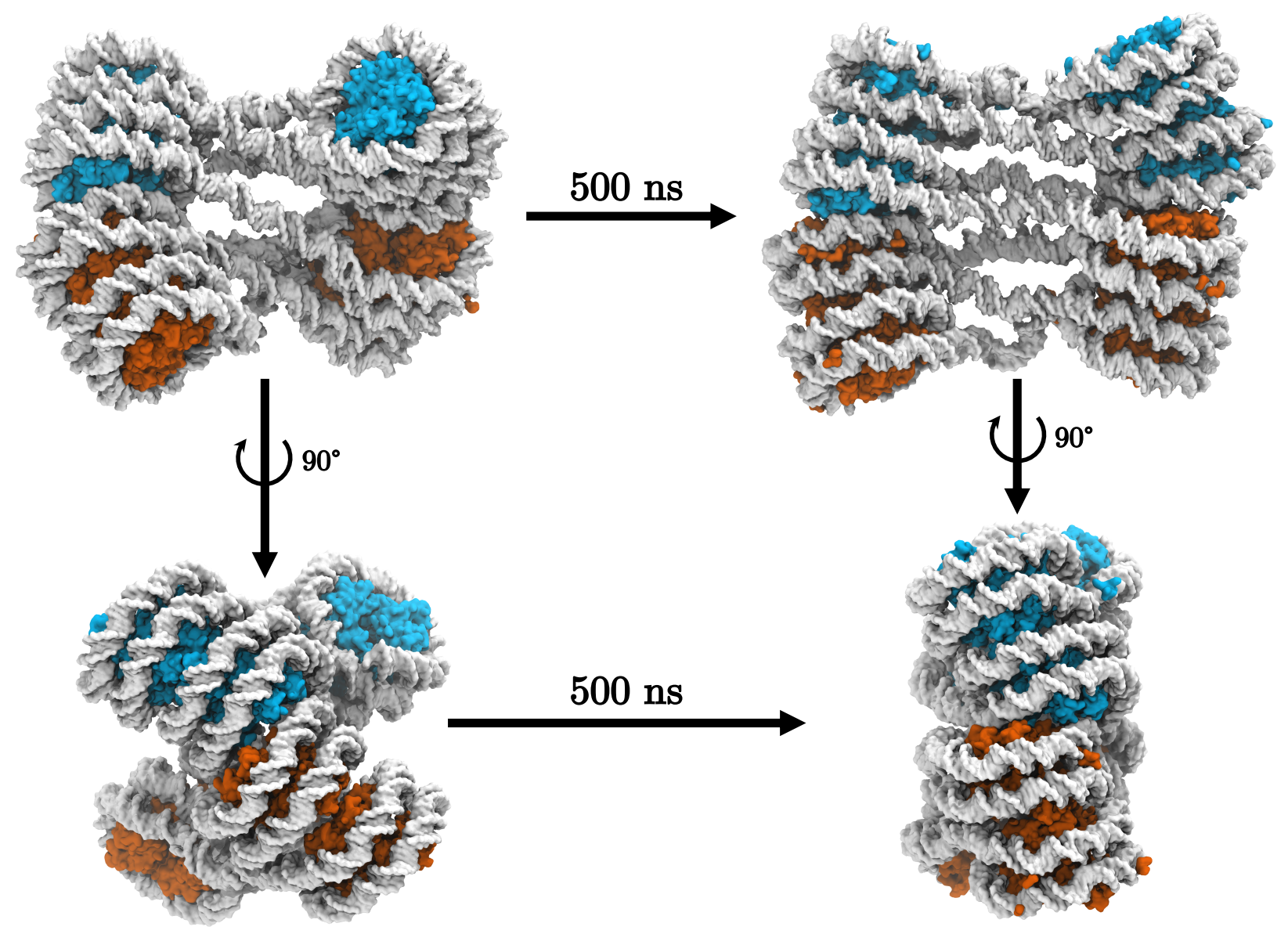

Figure 2: Shown are selected images from a system without the linker histone, at the beginning a simulation (left) and after $500 \mathrm{~ns}$ of production (right). The core histones in the poly-nucleosomes are colored to represent the different tetra-nucleosome sub-units as in Figure 1 .

\section{Results}

\section{Linker Histones Stabilize Tetra-Nucleosome Repeats}

Models of compact octa-nucleosome structures were generated through a combination of manual placement and flexible fitting of the $1 \mathrm{KX} 5$ nucleosome $\mathrm{e}^{\sqrt{79}}$ and $\mathrm{H} 1$ linker histone crystal structures ${ }^{\sqrt{75}}$ into the cryo-EM map by Song et al. (see Methods). $\frac{[22}{2}$ To quantify the configurations of these complexes, we took advantage of their double-helical like structures and measured the six canonical parameters of rise, twist, roll, tilt, shift, and slide, which are typically associated with DNA basepairs (see Methods for definitions). For each system there are three sets of tetra-nucleosomal sections, which for clarity we refer to as the top, middle, and bottom segments of the array and that contain nucleosomes one through four, three through six, and 

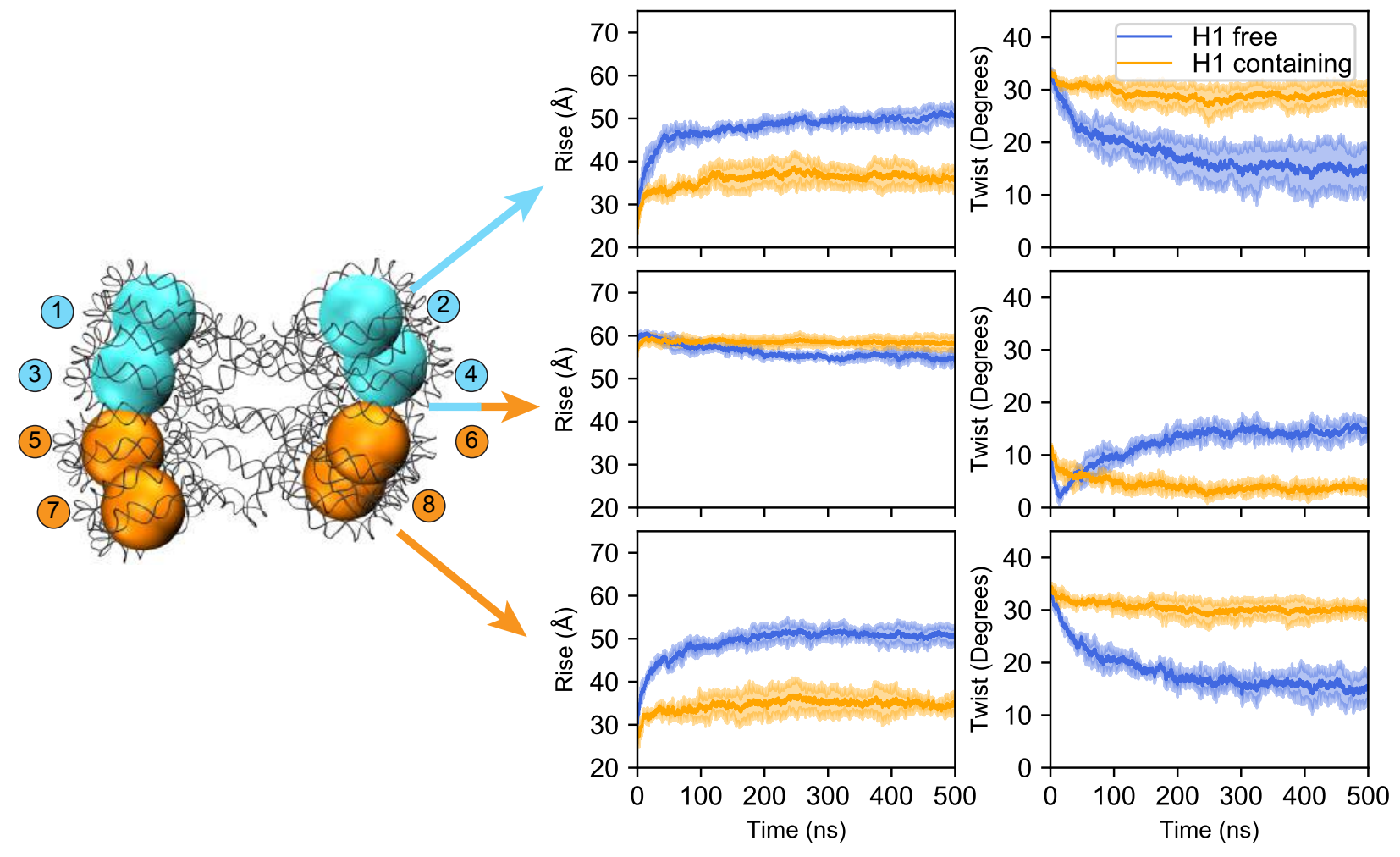

Figure 3: Nucleosomal rise and twist during simulations between the top, middle, and bottom four nucleosomes. Simulations with H1 (orange) maintain the initial stacked tetranucleosome structure, whereas simulations without H1 (blue) adopt a looser stacked conformation. Shown are the average and standard deviations (shaded regions) between the three simulations for each system. Colors of the octa-nucleosome array are to distinguish between tetra-nucleosome sub-units such as in Figures 1 and 2 Each nucleosome is designated with a number from 1 to 8 which is referenced throughout this manuscript.

five through eight respectively (Figures 2 and 3 ). Over our simulations, most of these metrics maintained values close to zero, with the exception of the inter-nucleosomal rise and twist which largely describe the observed large-scale conformational changes.

In each of the three $500 \mathrm{~ns}$ simulations we performed with H1, the rise and twist parameters remained similar to their initial values (middle and right of Figures 2, 3, and S1 S2). In particular, for both the bottom and top tetra-nucleosome segments (tetraNuc), the initial rise and twist of $\sim 27 \AA$ and $33^{\circ}$ were largely maintained, equilibrating at $\sim 35 \AA$ and $30^{\circ}$. In contrast, for the middle segment the initial and final rises were higher, with an average value of $58 \AA$, with a reduced twist that equilibrated from $14^{\circ}$ to $4^{\circ}$. This difference in values for the top and bottom array segments relative to the middle highlights the difference in the intra- and inter-tetraNuc structures: in a tetraNuc unit there is relatively little rise between nucleosomes (Nuc), as $\mathrm{Nuc}_{i}$ forms a tight packing interface with $\mathrm{Nuc}_{i+2}$ that creates a twist around the 
central fiber axis. Meanwhile, between tetraNuc structures the inter-nucleosomal packing is reduced and there is looser interface that has a higher rise and less twist around the helical axis. In addition, the minimal changes in these parameters during each simulation, and their reproducibility between each independent simulation (Figures S1 S2 demonstrates the stability on this stacked tetraNuc structure on the hundreds of nanoseconds timescales.

In contrast, in each of the three 500 ns simulations without H1, there were dramatic and irreversible changes in all measured rise and twist values which resulted in an elongated and less twisted array (Figures 2 and S6. Despite starting with values identical to H1 arrays, the stacked tetra-nucleosome structure was lost in the first $150 \mathrm{~ns}$, as is evidenced by the increase in rise of the top and bottom sections to $51 \AA$ and the decrease in twist to $15^{\circ}$. These values approach those of the middle array segment, which equilibrate to $53 \AA$ with an identical twist of $15^{\circ}$. This close agreement between the rise and twist for the middle with the top and bottom array segments demonstrates that the stacked tetraNuc structure is lost, as there is little physical difference between the structures of nucleosomes 1-4 and 5-8 with 3-6. This large conformational change is also demonstrated by the elevated root mean square deviation (RMSD) values for the $\mathrm{C}_{\alpha}$ and phosphate atoms, which ranged between 39 and $47 \AA$ for H1 lacking arrays, relative to a range of $15-18 \AA$ for H1 containing arrays (Figure S3).

\section{Greater Poly-Nucleosome Architecture Dictates Linker DNA Sampling}

Linker histones have a direct effect on the motions of linker DNA and nucleosomal DNA through favorable energetic interactions driven by electrostatic and Van der Waals forces. In a previous study, we emphasized the significance of these interactions by demonstrating how the linker histone binding pose, along with how their mere presence, can affect experimental results. ${ }^{75}$ Here, we translated these ideas to the context of poly-nucleosomal arrays by plotting the in- and out-of-nucleosomal-plane motions of both linker DNA arms in Figure 4 and Supplementary Figure S7, respectively. In general, removing linker histones from the array not only results in overall increased DNA sampling, but the development of novel linker DNA states. This is especially evident in the terminal nucleosomes, labelled Nuc 1, Nuc 2, Nuc 7, and Nuc 8 in Figures 4 and S7. Nucleosomes 2 and 7 presented the most structural distortion, which can be attributed to the drastic global change in conformation which occurred in the all simulations lacking linker histones.

To quantify these dynamics, the in- and out-of plane linker DNA motions were calculated and denoted as the $\alpha$ - and $\beta$ - angles, respectively (as inspired by Bednar et al., see Methods and Supporting Information for detailed definitions, Figures 5 and S4 . The $\alpha$-angles relate predominately to fluctuations in DNA breathing 
bioRxiv preprint doi: https://doi.org/10.1101/2020.09.20.305581; this version posted September 21, 2020. The copyright holder for this preprint (which was not certified by peer review) is the author/funder, who has granted bioRxiv a license to display the preprint in perpetuity. It is made available under aCC-BY-NC-ND 4.0 International license.

and ranged from $-112.2^{\circ}$ to $96.9^{\circ}$ with an average of $35.4^{\circ}$. Out-of-plane motions, or $\beta$-angles, ranged from $-56.4^{\circ}$ to $62.9^{\circ}$ and averaged $1.1^{\circ}$. An additional observation was that the entry and exit DNA arms of H1-absent nucleosomes had somewhat different probability distributions, which can be attributed to the asymmetric initial conformation within the poly-nucleosome array and the asymmetric nucleic acid sequence of Widom 601. While we previously illustrated that linker histone binding alters linker DNA fluctuations,
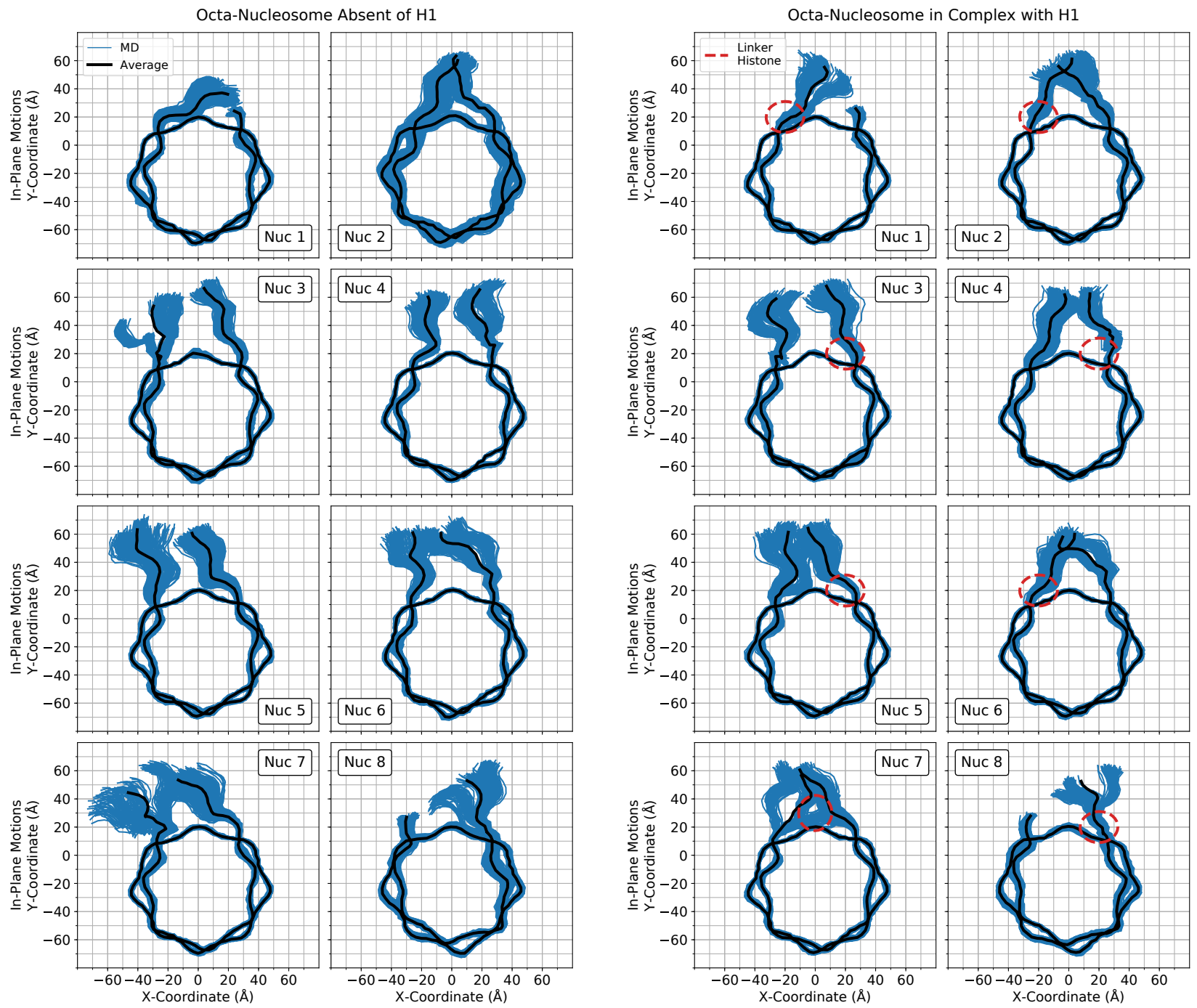

Figure 4: In-plane (top) DNA motions sampled by the octa-nucleosome arrays absent (left) and in complex (right) with the linker histone H1. Shown in blue are configurations sampled throughout the MD simulation (263 representative frames - every 4 ns of simulation time) while the average configuration is shown in black. For reference, the relative position of each nucleosome in its array is labeled in the corner of each graph. This label is consistent with the numbering in Figure 3 . Additionally, the approximate position of the linker histone is shown as a dashed-line red ellipse. Figures inspired by work from Shaytan et al ${ }^{[80}$ and single comparative nucleosome results were published previously. 

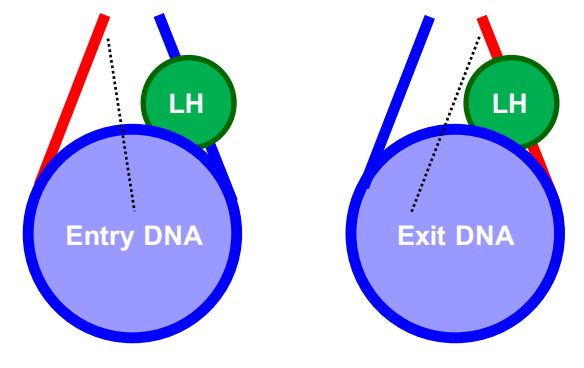

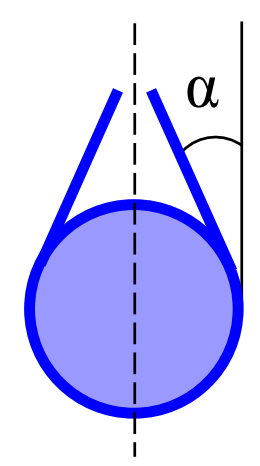

Front

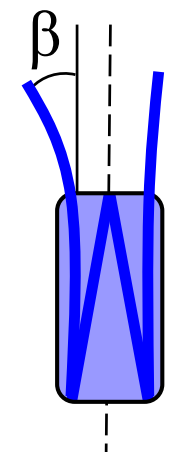

Side
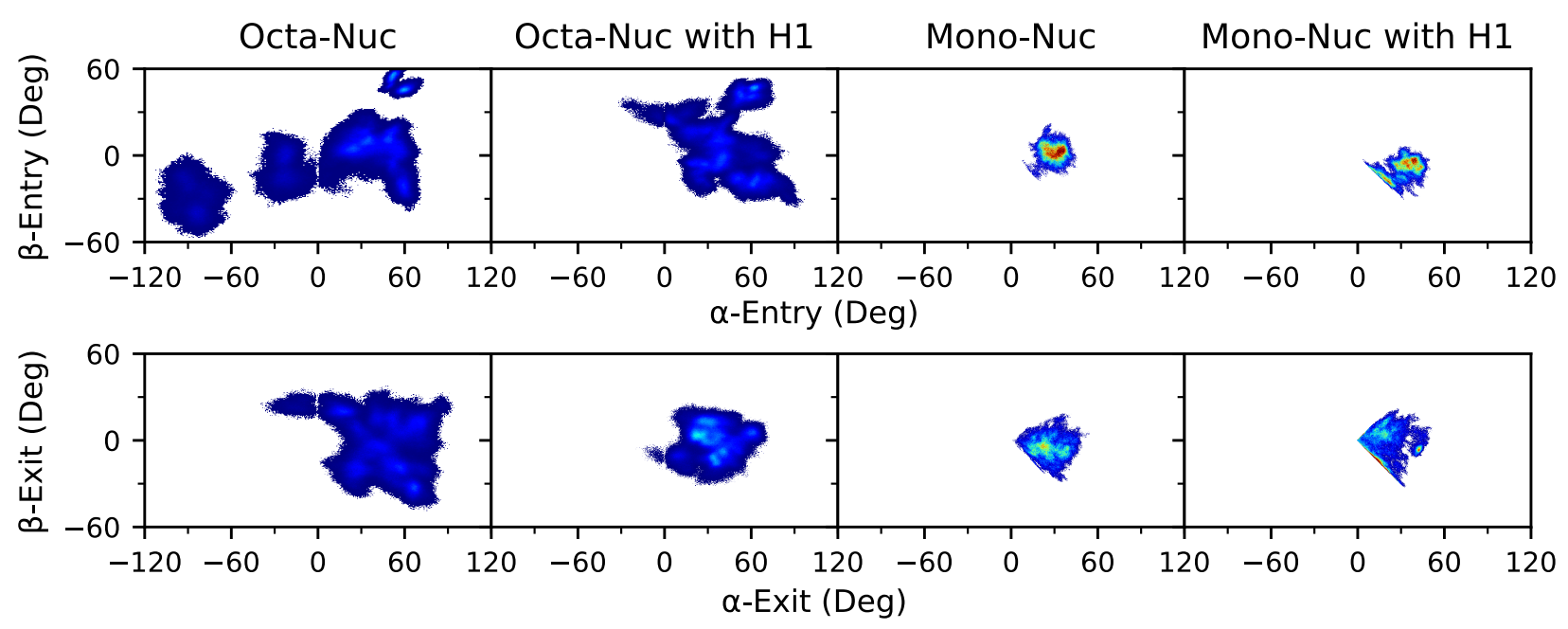

Figure 5: Comparison of DNA sampling for the entry (top plots) and exit (bottom plots) DNA segments for systems with and without H1. The $\alpha$-angles described in-plane motions, whereas $\beta$-angles describe outof-plane, as depicted in the diagrams on the to of the figure. For clarity, the entry- and exit-DNA segments are depicted in the top left diagram with the linker histone (LH), if present, in green. Density is represented as a gradient from blue (low density) to red (high density). Mono-nucleosome results are from previously published results. $\frac{75}{7}$

Figure 5 shows that those effects are more pronounced in poly-nucleosome systems. For example, the $\alpha$-Entry angle sampling range was reduced by $56.7^{\circ}$ when the octa-nucleosome is saturated with H1. However, this large reduction in sampling space is not present in the $\alpha$-Exit angles. This occurs because the majority of the structural strain within the compact array is distributed onto the Entry linker DNA. When the linker histone is no loner present, the distorted Entry DNA must endure the bulk of the conformational alleviation. In contrast to the mono-nucleosomes, the octa-nucleosome arrays sample a wider breadth of angles, particularly the $\alpha$-angle dimension. However, the individual nucleosomes that constitute the array do not readily transition throughout this entire phase space. More often, it is the case that each nucleosome 
Table 1: Jensen-Shannon distances (equation (4) for one dimensional probability distributions (Figure S5 of DNA motions between systems. For clarity, comparisons with low differences ( $\left.\mathrm{JS}_{\text {dist }}<0.20\right)$ are in blue, increased differences $\left(0.20<\mathrm{JS}_{\text {dist }}<0.40\right)$ are in green, high differences $(0.40<\mathrm{JS}$ dist $<0.60)$ are in orange, and very high differences $\left(0.60<\mathrm{JS}_{\text {dist }}<1.00\right)$ in red. The lower numerical values correspond to a greater similarity in probability distributions, whereas higher numerical values correspond to a greater dissimilarity. Two identical distributions will produce a Jensen-Shannon distance of 0.00 , whereas distributions that do no share any phase space commonality will result in 1.00 .

\begin{tabular}{|c|c|c|c|c|c|c|}
\hline & \multicolumn{3}{|c|}{$\alpha$-Entry } & \multicolumn{3}{|c|}{$\alpha$-Exit } \\
\hline & $\begin{array}{c}\text { MonoNuc } \\
\text { with H1 }\end{array}$ & OctaNuc & $\begin{array}{l}\text { OctaNuc } \\
\text { with H1 }\end{array}$ & $\begin{array}{c}\text { MonoNuc } \\
\text { with H1 }\end{array}$ & OctaNuc & $\begin{array}{l}\text { OctaNuc } \\
\text { with } \mathrm{H} 1\end{array}$ \\
\hline \multirow{5}{*}{$\begin{array}{l}\text { MonoNuc } \\
\text { MonoNuc with H1 } \\
\text { OctaNuc }\end{array}$} & 0.35 & 0.69 & 0.64 & 0.35 & 0.62 & 0.36 \\
\hline & & 0.63 & 0.54 & & 0.67 & 0.54 \\
\hline & & & 0.42 & & & 0.41 \\
\hline & \multicolumn{3}{|c|}{$\beta$-Entry } & \multicolumn{3}{|c|}{$\beta$-Exit } \\
\hline & $\begin{array}{l}\text { MonoNuc } \\
\text { with H1 }\end{array}$ & OctaNuc & $\begin{array}{l}\text { OctaNuc } \\
\text { with H1 }\end{array}$ & $\begin{array}{c}\text { MonoNuc } \\
\text { with H1 }\end{array}$ & OctaNuc & $\begin{array}{l}\text { OctaNuc } \\
\text { with H1 }\end{array}$ \\
\hline \multirow{3}{*}{$\begin{array}{l}\text { MonoNuc } \\
\text { MonoNuc with H1 } \\
\text { OctaNuc }\end{array}$} & 0.72 & 0.53 & 0.64 & 0.30 & 0.59 & 0.36 \\
\hline & & 0.69 & 0.58 & & 0.44 & 0.15 \\
\hline & & & 0.39 & & & 0.46 \\
\hline
\end{tabular}

will exist as a single stable, but independent, state unable to sample much outside of its respective potential well. Despite the apparent increased sampling of angles within the array, there is an inherent entropic cost for each individual nucleosome as oppose to existing solitary in solution.

Although mono-nucleosomes had more available conformational freedom, linker DNA in poly-nucleosomes sample a broader spectrum of states throughout the simulations, which we attribute to the strained nature of nucleosome arrays biasing linker DNA into ordinarily unattainable states, as observed in Figure 4 To further quantify these differences, we calculated Jensen-Shannon distances (JS dist $)$ (Table 1), based on the Jensen-Shannon divergence (JSD), between the one-dimensional probability distributions displayed in Figure S4. Values closer to zero correspond to a greater similarity in probability distributions, whereas values approaching 1.0 correspond to a greater dissimilarity. The $\mathrm{JS}_{\text {dist }}$ values generally exhibit a stark contrast between mono-nucleosome and octa-nucleosome systems with values often above 0.50 , although with some exceptions. In particular, $\alpha / \beta$-angles of the Exit DNA (in contact with H1) sample a much more similar phase space than the Entry DNA angles. Additionally, highlighted by a $\mathrm{JS}_{\text {dist }}$ of 0.72 , binding of the H1 severely alters sampling of $\beta$-Entry angles in mono-nucleosomes and thus presenting an extreme case for which to compare poly-nucleosome systems. 

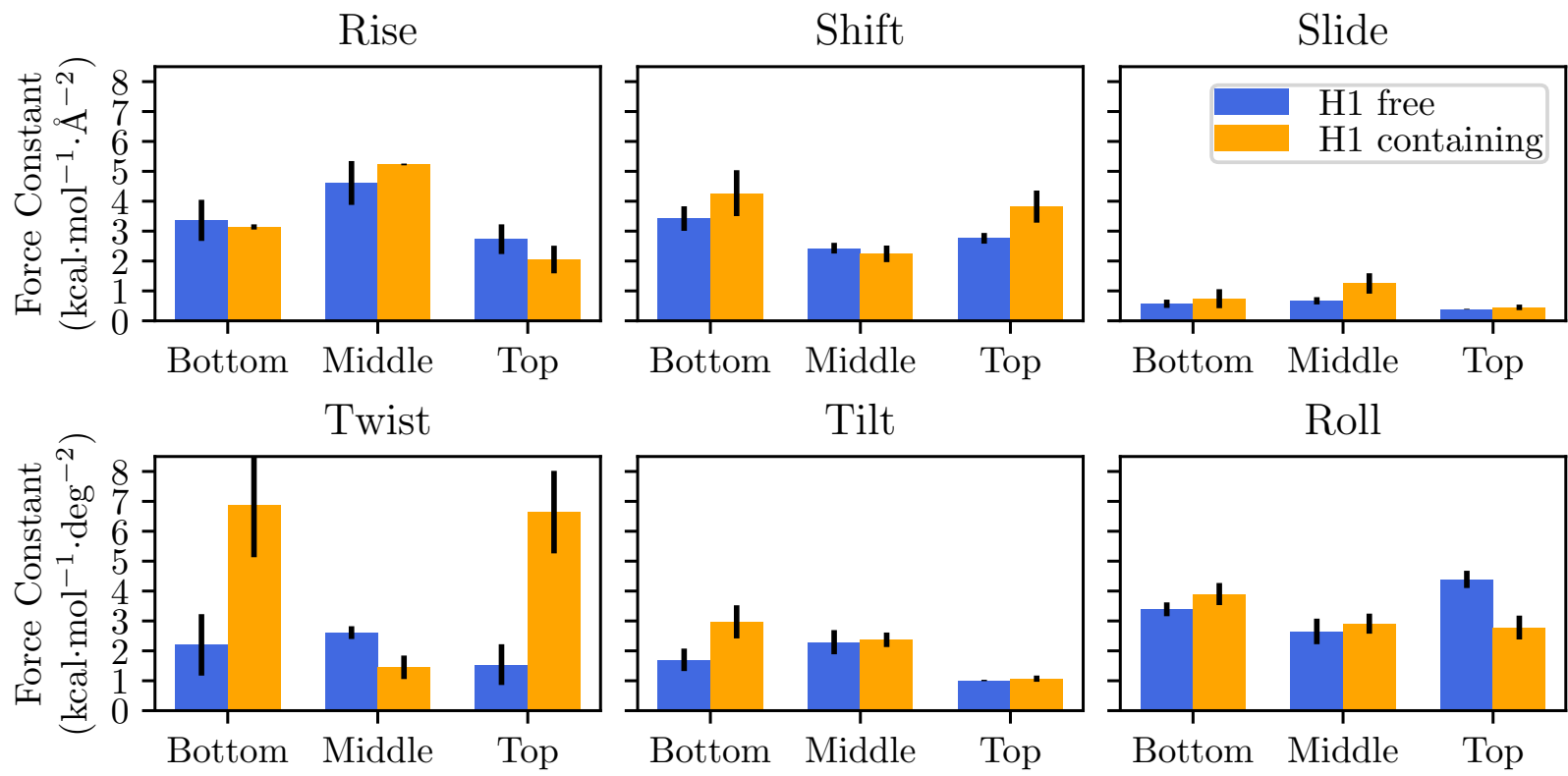

Figure 6: Force constants of helical parameters for the Bottom, Middle, and Top tetra-nucleosomal structures (as defined in Figure S1) and reported in Tables S1 and S2. Error bars represent the standard error of the mean computed from all three simulations.

\section{Linker Histones Lead to Stiffer Nucleosome Arrays}

To characterize the effects of linker histones on the flexibility of compact nucleosomal arrays, the local elastic properties of these systems were computed based on the helical parameter covariance matrices (see Methods for details). For each of the diagonal elements in these matrices, the associated force constants were equal or higher for $\mathrm{H} 1$ containing arrays as compared to $\mathrm{H} 1$ free arrays (Figure 6). However, in many cases the stiffness, and the difference between the H1 free and containing systems, was dependent on whether inter- or intra-tetra-nucleosome units were considered. For example, in consideration of the rise parameter, in H1-free arrays all of these elastic constants had values that were approximately equal to one another (within the standard error). In H1-containing arrays the stiffness parameters were similar, however, given the smaller standard errors we are able to conclude that the inter-nucleosome rise stiffness is sightly higher than the intra-nucleosome stiffness. Other parameters, such as slide shift, tilt, and roll, displayed a similar trend that any difference between the stiffness parameters were small, and close to the standard errors. In contrast, linker histones created a significant increase in the stiffness of the twist within tetra-nucleosome units, but did not influence the force constants between them, suggesting that stacked tetra-nucleosome units impart resistance in poly-nucleosomal arrays via torsional stress.

While the on-diagonal elements of the stiffness matrices are the force constants for the canonical helical 
(a.) $\mathrm{H} 1$ free

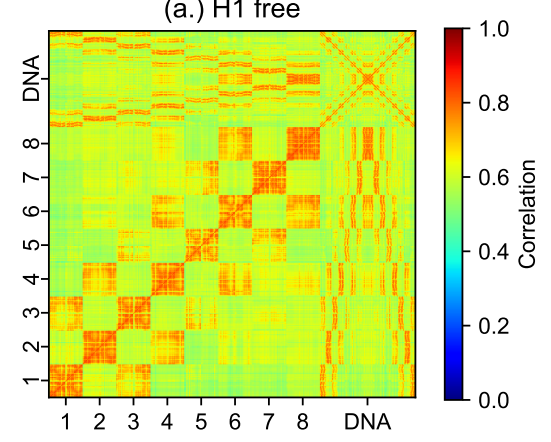

(b.) $\mathrm{H} 1$ containing

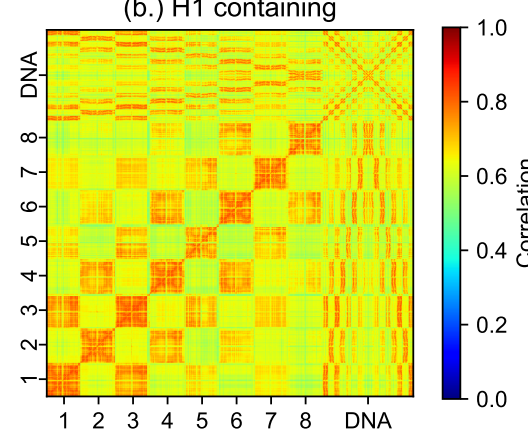

(c.) $\mathrm{H} 1$ containing- $\mathrm{H} 1$ free

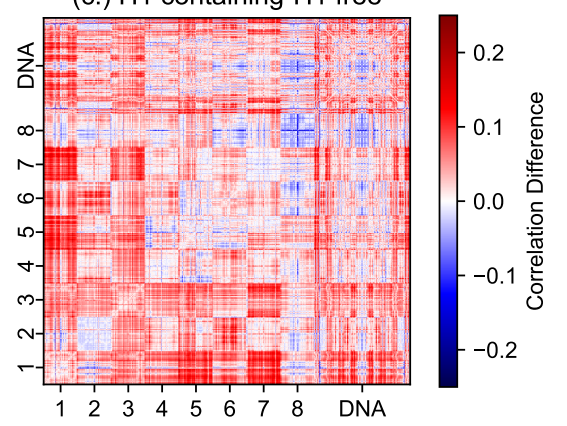

Figure 7: Inter-residue correlations for systems lacking (a.) and containing (b.) H1. H1 increases system correlations, notably through increased correlations in stacked nucleosomes, as shown in the difference between system with and without H1 (c.).

parameter, the off-diagonal elements correspond to the coupling between these parameters (Tables S1-S2). The majority of these elements are small and within the standard error of zero, indicating that these degrees of freedom are largely uncorrelated from one another. In contrast, the twist-rise coupling is significant and shows a pattern similar to the twist force constants: without $\mathrm{H} 1$ the coupling constants range from $1.60 \pm$ 0.51 to $2.95 \pm 0.24 \mathrm{kcal} \cdot \mathrm{mol}^{-1} \cdot \AA^{-1} \cdot \mathrm{deg}^{-1}$, with the highest being for the middle segment of the octa-nucleosome array. In contrast, there is a more significant difference with $\mathrm{H} 1$, where the bottom and top tetra-nucleosome segments contain coupling constants of $3.92 \pm 0.45$ and $3.08 \pm 0.69 \mathrm{kcal} \cdot \mathrm{mol}^{-1} \cdot \AA^{-1} \cdot \mathrm{deg}^{-1}$, and the middle has a significantly reduced value of $1.21 \pm 0.24 \mathrm{kcal} \cdot \mathrm{mol}^{-1} \cdot \AA^{-1} \cdot \mathrm{deg}^{-1}$. This further points to the increased rigidity within linker histone stabilized tetra-nucleosome units, and the relative looseness in these arrays between them. In addition, the positive values observed for all twist-rise coupling constants show that these arrays contract upon overtwisting, which is in line with what one would expect from models of simple helical elastic polymers but is contrary to DNA which elongates when overtwisted. $\frac{8182}{82}$

\section{Linker Histones Create Long Range Correlations}

Having established that linker histones create stiffer nucleosomal arrays, we sought to understand the implications for larger scale dynamical properties. We therefore performed a generalized correlation analysis on each system to determine the pairwise correlations between each DNA base and protein residue in the system. $\frac{83}{83}$ The average H1 free correlation matrix shows the expected behavior that within each nucleosome there is a high correlation, as individual nucleosomes are highly rigid on the nanosecond timescale (see red square in Figure 77). In addition, core histones are highly correlated with the DNA which wraps around it, as evidenced by the red patterns at the top and right side of these figures, and DNA bases are highly 
correlated with the base they are paired with, as shown by the "X" mark in the upper right hand corner. More interestingly, individual nucleosomes are highly correlated with nucleosomes that stack directly above or below them. That is, $\mathrm{Nuc}_{i}$ is highly correlated with $\mathrm{Nuc}_{i+2}$ and $\mathrm{Nuc}_{i-2}$. In contrast, nucleosomes did not have a large correlation with their adjacent nucleosome, as the $\mathrm{Nuc}_{i}$ and $\mathrm{Nuc}_{i+1}$ correlations were relatively low.

Upon the addition of linker histones, the overall pattern of strong intra-nucleosomal and local nucleosome/DNA correlations remained (Figure 7b). In addition, the majority of correlations were enhanced, as evidenced in the difference map between the correlations in the H1 containing and free systems (Figure 7 ). Of particular interest are the stronger correlations between all of the odd numbered and even numbered nucleosomes, that is between nucleosomes $1,3,5$, and 7 and 2,4,6, and 8. This suggests that in the more compact and stiffer arrays induced by linker histones, correlations are able to propagate throughout each side of these two-start zig-zag arrays much further then they can without linker histones.

\section{Discussion}

Here, we have used conventional MD simulations to probe the effects of linker histone binding on an octanucleosome model of the chromatin fiber. We calculated nucleosomal helical parameters quantifying a global conformational shift that demonstrate the importance of $\mathrm{H} 1$ in the structural stability of chromatin fibers. Moreover, we have captured a physical phenomenon that has been rarely observed experimentally 51 - the helical untwisting of the poly-nucloeosme array. The most probable explanation for this occurrence is the lack of strong protein-DNA interactions provided by the linker-histone/DNA motif. The chromatin fiber is highly compact and very strained by our observations. The saturated binding of linker histones to the linker DNA stabilizes the system and prevents it from untwisting. Presumably, this untwisting effect could be mimicked by a decrease in salt concentration. In fact, Garcia-Saez et al. presented an untwisted nucleosome array model based on cryo-EM data of arrays under low-salt conditions. Interestingly, this phenomenon was not hindered by the presence of the linker histone, but occurs readily in saturated arrays. One potential explanation for this discrepancy is that the Garcia-Saez et al. poly-nucleosome array exhibited a low packing density resulting in inherently less inter-nucleosome protein-DNA interactions. This can be attributed to longer linker DNA length between nucleosomes (50 bp versus $30 \mathrm{bp}$ ) and the more rigid on-dyad binding mode. As shown in Figures 4 and 5 , our more compact model requires more diverse sampling of the linker DNA, which was previously shown to be hindered upon on-dyad binding. ${ }^{75}$ A compact chromatin fiber with 
H1 bound on-dyad would be more strained than its off-dyad bound counterpart and easily perturbed upon a reduction in ionic strength, as observed by Garcia-Saez et al.

By altering linker DNA dynamics, linker histones inherently inhibit transcription and promote the compaction of chromatin fibers. Our simulations have shown how substantial an impact the linker histones have on the global chromatin compaction. This effect is highlighted by the dramatic reduction in sampling space upon H1 binding. Once bound, the linker histone increases the rigidity of the entire system, as was further emphasized by the increase in correlation throughout the fiber (Figure 7). Furthermore, the disparity in sampling between mono- and poly-nucleosomes is extensive. We accredit this observation to the unique structure of the chromatin fiber. In mono-nucleosomes, linker DNA is generally free to move about in solution, unless bound to a linker histone. However, in poly-nucleosome arrays each nucleosome must adopt a specific conformation to alleviate strain on the entire system. Here, we stress caution when studying mono-nucleosomes and deducing conclusions about their dynamics. The Jensen-Shannon distances in Table 1 highlights the vast dissimilarities between the two systems, specifically with in-plane linker DNA motions, and why results from mono-nucleosome studies, especially related to DNA, may not be transferable when describing the greater chromatin architecture.

The octa-nucleosome system studied here is composed of two distinct, although attached, tetra-nucleosome sub-units, as illustrated throughout this manuscript by light-blue and orange colored core histones (Figures 1. 3, 2, and S6 . Using force constants derived from the helical parameters, we found that linker histones impart increased torsional stress within these tetra-nucleosome units while slightly decreasing it between units. Interestingly, linker histones between tetra-nucleosome sub-units are quite close proximity to one another and have been speculated to interact, ${ }^{22151}$ giving rise to potential sites for post-translational modifications. $\frac{\sqrt{84}, 87}{87}$ Our calculations show only a few inter-linker histone contacts, specifically between linker histones associated with $\mathrm{Nuc}_{4}$ and $\mathrm{Nuc}_{6}$ (Figures $\mathrm{S} 8$ and $\mathrm{S} 9$. Unfortunately, the presence of these interactions did not contribute to the overall stiffness of our model. This is evident by the aforementioned force constants which show a decrease in torsional stress between tetra-nucleosome sub-units upon H1 binding. Therefore, by our observations, inter-linker histone interactions do not significantly contribute to chromatin compaction in the octa-nucleosome model studied here. However, it should be stated that this model included only the globular domain of each linker histone and not the N- and C-terminal tails, known to interact with H3 tails to facilitate binding, $\frac{88}{18}$ translating to an examination of localized interactions. The inclusion of these motifs may lead to more interactions with other linker histones, linker DNA, and/or nucleosomes, resulting in increased chromatin fiber compaction and rigidity. 
In a recent comprehensive study, Perišić et al. used meso-scale modeling to demonstrate the extent to which linker histone binding modes and variants affect chromatin compaction. ${ }^{89}$ They were able to connect existing ideas suggesting that combinations of on- and off-dyad binding result in varying levels of compaction on a spectrum between condensed ${ }^{[22}$ and uncondensed ${ }^{[00}$ arrays, respectively. Their work provided strong reinforcement that shifts between these two states are directly associated with a shift in linker histone binding mode, 51 a sentiment which we share. ${ }^{75}$ Here, we quantified the effects of linker histones on condensation using various metrics from an atomistic perspective. Furthermore, this work demonstrates that chromatin can experience large conformational transitions in timescales of under a microsecond, which is well under the time it may take to expose nucleosomes for transcription and DNA repair. 91,93

\section{Materials and Methods}

\section{System Construction}

Core histones and the asymmetric Widom 601 DNA were modelled based on the 1KX5 crystal structure (resolution $1.94 \AA^{(79}$ ). Missing residues and nucleotides were added using Modeller via the Chimera graphical

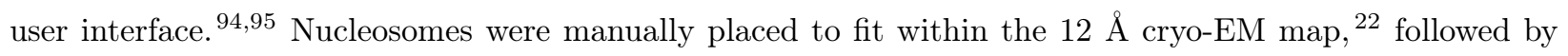
30 bp of linker DNA between them, which was built using the Nucleic Acids Builder (NAB) module within AmberTools18 software package. ${ }^{96}$ From here, rigid docking was performed using the Colores module of Situs, ${ }^{97 / 98}$ which was followed by flexible docking using internal coordinates normal mode analysis (iMOD). .99 Finally, we built, placed, and validated the linker histone binding mode within the octa-nuclesome array using methods detailed in our previous work. $\frac{[75}{6}$ When solvated, these systems were approximately 2,757,000 atoms.

\section{Molecular Dynamics simulations}

All systems were prepared and simulated using the GROMACS 2016.4 software package. ${ }^{100}$ Each system was solvated in a TIP3P water box extending at least $10 \AA$ from the solute.101102 Using Joung-Cheatham

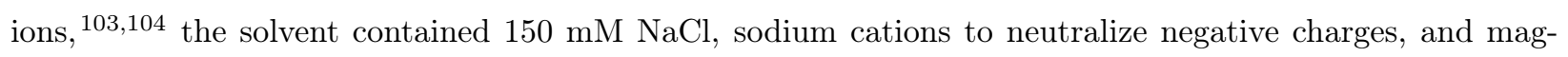
nesium ions that replaced the manganese ions in the 1KX5 crystal structure. Only magnesium ions in the DNA grooves were included, whereas those located close to the the linker histones binding locations were excluded so as to not interfere with LH-DNA interactions. The AMBER14SB and BSC1 force fields were used for protein and DNA interactions, respectively. $[105 / 106$ A cutoff distance of $10.0 \AA$ with a switching function beginning at $8.0 \AA$ was used for nonbonded interactions, and long range electrostatics were treated 
with particle mesh Ewald calculations. $\frac{107}{}$ Systems were minimized for 10,000 steps, and then equilibrated for $100 \mathrm{ps}$ at constant volume and temperature and for $1 \mathrm{~ns}$ at constant pressure and temperature. Production simulations were carried out for $500 \mathrm{~ns}$ in the NPT ensemble, using a Parrinello-Rahman barostat 108 with a time constant of $1.0 \mathrm{ps}$ to control the pressure and a Nosé-Hoover thermostat at $300 \mathrm{~K}$ with a time constant 0.5 ps. Electrostatic interactions were treated with the Particle-Mesh Ewald (PME) method ${ }^{107}$ and $10 \AA$ cut-off. Simulations were conducted on two systems: one with the linker histone bound to the DNA of each nucleosome and one without the presence of H1. Each simulation was run in triplicate for 500 ns with a 2 fs timestep using resources provided by the Extreme Science and Engineering Discovery Environment (XSEDE) 109

\section{Analysis}

\section{Helical Parameters}

The local double helical structure of the chromatin fiber was quantified based upon three translational (rise, shift, and slide) and three rotational (twist, tilt, and roll) parameters. ${ }^{77}$ In analogy with DNA structure, a "base pair" was defined as two nucleosomes that were in the same z-plane with one another, where the z-axis is defined as the principal fiber axis. For each nucleosome pair, the local x-axis was defined as a vector from the center of mass of the DNA phosphate atoms of NCP $i$ to the center of mass of the DNA phosphate atoms of NCP $i+1$. The z-axis was defined as the average of the third principal axis of inertia of NCPs $i$ and $i+1$, as computed with gromacs, and the y-axis was computed as the vector perpendicular to these two vectors. Following the definition of these "base-pair" axis, the algorithm outlined by Lu et al. was used to compute the rise, shift, slide, twist, tilt, and roll. $\frac{76}{76}$

To compute the elastic force constants, a harmonic approximation was made for each of the basepair parameters, such that the internal energy of the fiber is estimated by:

$$
U(w)=\frac{1}{2}(w-\hat{w}) \cdot K(w-\hat{w})
$$

where $w$ is the vector of inter-base pair parameters, $\hat{w}$ is their mean, and $K$ a stiffness matrix. $\frac{110}{\text { Given }}$ small fluctuations, the equipartion theorem can be used to construct $K$ from the inverse of the covariance matrix, C:

$$
K=k_{b} T C^{-1}
$$


where $k_{b}$ is Boltzmann's constant, and $\mathrm{T}$ the system temperature. Terms along the diagonal of $\mathrm{K}$ are the individual parameter force constants, and off-digonal terms represent the parameter coupling constants. Error bars were computed by calculating the stiffness matrices for each of the three simulations and taking the standard error of the mean.

\section{Generalized Correlation}

Mutual-information based generalized correlation methods were employed to capture non-collinear correlations between residue pairs to describe both linear and non-linear coupled motions. Results were computed using the g_correlation plugin for GROMACS/3.3. ${ }^{83100 \mid 111}$ The first $150 \mathrm{~ns}$ of simulation time was allotted for equilibration while trajectories were analyzed every $50 \mathrm{ps.} \mathrm{Analyses} \mathrm{were} \mathrm{performed} \mathrm{only} \mathrm{on} \mathrm{the} \mathrm{protein}$ $\mathrm{C}_{\alpha}$ and the nucleotide $\mathrm{C} 1$ ' heavy atoms.

\section{Linker DNA Dynamics}

The linker DNA in- and out-of nucleosomal plane motions were quantified to describe the linker DNA motions. To define the plane, the nucleosomal DNA was divided into four quadrants and the center of mass of the C1' atoms within the two quadrants located distal from the linker DNA were used for two points, while the third point was defined as the $\mathrm{C}^{\prime}$ ' center of mass of bases 83 and 250 which are located approximately on the dyad axis (see previous work ${ }^{75}$ for details). The linker DNA vectors were defined as the C1' center of mass of the base pairs at the origin of the linker DNA (bases 20-315 and 148-187) and terminal base pairs (bases 1-354 and 177-178), respectively. The $\alpha$-angles were defined as in-plane and the $\beta$-angles were defined as out-of-plane motions of this vector. Positive $\alpha$-angles were defined as inward motions towards the dyad axis while positive $\beta$-angles were defined as outward motions away from the nucleosomal-plane. For reference, the angles shown in Figure 5 are positive.

The change in linker DNA sampling between H1-bound and -free systems were computed using two metrics, the Kullback-Leibler ${ }^{112}$ (KLD) and Jensen-Shannon divergences (JSD), $\stackrel{113[114}{ }$ respectively:

$$
\begin{gathered}
D_{K L}(P \| Q)=-\sum_{x \in X} P(x) \log _{2}\left(\frac{Q(x)}{P(x)}\right) \\
D_{J S}(P \| Q)=\frac{1}{2} D_{K L}(P \| M)+\frac{1}{2} D_{K L}(Q \| M)
\end{gathered}
$$


where,

$$
M=\frac{1}{2}(P(x)+Q(x))
$$

where, in equation (4), $Q(x)$ is the normalized reference distribution and $P(x)$ is the normalized data set. In equation (4), the JSD gives equal weight to $Q(x)$ and $P(x)$ by calculating their KLD with respect to an average distribution, $M$ in equation (5). With these measures, we are comparing two probability distributions and thus employ a base 2 logarithm as shown in equation (3). Due to its symmetric nature, the square root of the JSD can be used as a true mathematical metric known as the Jensen-Shannon distance ${ }^{115}$-117 which is how we have reported it in this study.

\section{Author Contributions}

F.R.R. ran the simulations of the poly-nucleosome array. J.W. and D.C.W. ran the analyses and prepared the manuscript. All authors contributed to the design of this project.

\section{Acknowledgments}

Work in the Wereszczynski group is funded by the National Science Foundation [CAREER-1552743] and the National Institutes of Health [1R35GM119647]. This work used the Extreme Science and Engineering Discovery Environment, which is supported by the National Science Foundation [ACI-1053575]. 


\section{References}

[1] Kornberg, R. D. Chromatin structure: a repeating unit of histones and DNA. Science 1974, 184, 868-871.

[2] McGinty, R. K.; Tan, S. Nucleosome structure and function. Chem. Rev. 2015, 115, 2255-2273.

[3] Olins, A. L.; Olins, D. E. Spheroid chromatin units (v bodies). Science 1974, 183, 330-332.

[4] Kaplan, N.; Moore, I. K.; Fondufe-Mittendorf, Y.; Gossett, A. J.; Tillo, D.; Field, Y.; LeProust, E. M.; Hughes, T. R.; Lieb, J. D.; Widom, J. et al. The DNA-encoded nucleosome organization of a eukaryotic genome. Nature 2009, 458, 362-366.

[5] Luger, K.; Mader, A. W.; Richmond, R. K.; Sargent, D. F.; Richmond, T. J. Crystal structure of the nucleosome core particle at $2.8 \AA$ resolution. Nature 1997, 389, 251-260.

[6] Baldi, S.; Korber, P.; Becker, P. B. Beads on a string-nucleosome array arrangements and folding of the chromatin fiber. Nat. Struct. Mol. Biol. 2020, 27, 109-118.

[7] Wiese, O.; Marenduzzo, D.; Brackley, C. A. Nucleosome positions alone can be used to predict domains in yeast chromosomes. Proc. Natl. Acad. Sci. U.S.A. 2019, 116, 17307-17315.

[8] Bass, M. V.; Nikitina, T.; Norouzi, D.; Zhurkin, V. B.; Grigoryev, S. A. Nucleosome spacing periodically modulates nucleosome chain folding and DNA topology in circular nucleosome arrays. J. Biol. Chem. 2019, 294, 4233-4246.

[9] Pusarla, R. H.; Vinayachandran, V.; Bhargava, P. Nucleosome positioning in relation to nucleosome spacing and DNA sequence-specific binding of a protein. FEBS J. 2007, 274, 2396-2410.

[10] Noll, M.; Kornberg, R. D. Action of micrococcal nuclease on chromatin and the location of histone H1. J. Mol. Biol. 1977, 109, 393-404.

[11] Blank, T. A.; Becker, P. B. Electrostatic mechanism of nucleosome spacing. J. Mol. Biol. 1995, 252, 305-313.

[12] Zhang, T.; Zhang, W.; Jiang, J. Genome-Wide Nucleosome Occupancy and Positioning and Their Impact on Gene Expression and Evolution in Plants. Plant Physiol. 2015, 168, 1406-1416.

[13] Rodríguez-Campos, A.; Shimamura, A.; Worcel, A. Assembly and properties of chromatin containing histone H1. J. Mol. Biol. 1989, 209, 135-150. 
[14] Garcia-Ramirez, M.; Dong, F.; Ausio, J. Role of the histone "tails" in the folding of oligonucleosomes depleted of histone H1. J. Biol. Chem. 1992, 267, 19587-19595.

[15] Tremethick, D. J.; Drew, H. R. High mobility group proteins 14 and 17 can space nucleosomes in vitro. J. Biol. Chem. 1993, 268, 11389-11393.

[16] Robinson, P. J.; Fairall, L.; Huynh, V. A.; Rhodes, D. EM measurements define the dimensions of the "30-nm" chromatin fiber: evidence for a compact, interdigitated structure. Proc. Natl. Acad. Sci. U.S.A. 2006, 103, 6506-6511.

[17] Finch, J. T.; Klug, A. Solenoidal model for superstructure in chromatin. Proc. Natl. Acad. Sci. U.S.A. 1976, 73, 1897-1901.

[18] Worcel, A.; Strogatz, S.; Riley, D. Structure of chromatin and the linking number of DNA. Proc. Natl. Acad. Sci. U.S.A. 1981, 78, 1461-1465.

[19] Williams, S. P.; Athey, B. D.; Muglia, L. J.; Schappe, R. S.; Gough, A. H.; Langmore, J. P. Chromatin fibers are left-handed double helices with diameter and mass per unit length that depend on linker length. Biophys. J. 1986, 49, 233-248.

[20] Dorigo, B.; Schalch, T.; Kulangara, A.; Duda, S.; Schroeder, R. R.; Richmond, T. J. Nucleosome arrays reveal the two-start organization of the chromatin fiber. Science 2004, 306, 1571-1573.

[21] Schalch, T.; Duda, S.; Sargent, D. F.; Richmond, T. J. X-ray structure of a tetranucleosome and its implications for the chromatin fibre. Nature 2005, 436, 138-141.

[22] Song, F.; Chen, P.; Sun, D.; Wang, M.; Dong, L.; Liang, D.; Xu, R. M.; Zhu, P.; Li, G. Cryo-EM study of the chromatin fiber reveals a double helix twisted by tetranucleosomal units. Science 2014, 344, $376-380$.

[23] Ekundayo, B.; Richmond, T. J.; Schalch, T. Capturing Structural Heterogeneity in Chromatin Fibers. J. Mol. Biol. 2017, 429, 3031-3042.

[24] Grigoryev, S. A.; Arya, G.; Correll, S.; Woodcock, C. L.; Schlick, T. Evidence for heteromorphic chromatin fibers from analysis of nucleosome interactions. Proc. Natl. Acad. Sci. U.S.A. 2009, 106, 13317-13322. 
[25] Greulich, K. O.; Wachtel, E.; Ausio, J.; Seger, D.; Eisenberg, H. Transition of chromatin from the "10 $\mathrm{nm}$ " lower order structure, to the "30 nm" higher order structure as followed by small angle X-ray scattering. J. Mol. Biol. 1987, 193, 709-721.

[26] Thoma, F.; Koller, T.; Klug, A. Involvement of histone H1 in the organization of the nucleosome and of the salt-dependent superstructures of chromatin. J. Cell Biol. 1979, 83, 403-427.

[27] Makarov, V. L.; Dimitrov, S. I.; Petrov, P. T. Salt-induced conformational transitions in chromatin. A flow linear dichroism study. Eur. J. Biochem. 1983, 133, 491-497.

[28] Gerchman, S. E.; Ramakrishnan, V. Chromatin higher-order structure studied by neutron scattering and scanning transmission electron microscopy. Proc. Natl. Acad. Sci. U.S.A. 1987, 84, 7802-7806.

[29] Ghirlando, R.; Felsenfeld, G. Hydrodynamic studies on defined heterochromatin fragments support a 30-nm fiber having six nucleosomes per turn. J. Mol. Biol. 2008, 376, 1417-1425.

[30] Eltsov, M.; Maclellan, K. M.; Maeshima, K.; Frangakis, A. S.; Dubochet, J. Analysis of cryo-electron microscopy images does not support the existence of 30-nm chromatin fibers in mitotic chromosomes in situ. Proc. Natl. Acad. Sci. U.S.A. 2008, 105, 19732-19737.

[31] Fussner, E.; Strauss, M.; Djuric, U.; Li, R.; Ahmed, K.; Hart, M.; Ellis, J.; Bazett-Jones, D. P. Open and closed domains in the mouse genome are configured as 10-nm chromatin fibres. EMBO Rep. 2012, 13, 992-996.

[32] Gan, L.; Ladinsky, M. S.; Jensen, G. J. Chromatin in a marine picoeukaryote is a disordered assemblage of nucleosomes. Chromosoma 2013, 122, 377-386.

[33] Nishino, Y.; Eltsov, M.; Joti, Y.; Ito, K.; Takata, H.; Takahashi, Y.; Hihara, S.; Frangakis, A. S.; Imamoto, N.; Ishikawa, T. et al. Human mitotic chromosomes consist predominantly of irregularly folded nucleosome fibres without a 30-nm chromatin structure. EMBO J. 2012, 31, 1644-1653.

[34] Ou, H. D.; Phan, S.; Deerinck, T. J.; Thor, A.; Ellisman, M. H.; O’Shea, C. C. ChromEMT: Visualizing 3D chromatin structure and compaction in interphase and mitotic cells. Science 2017, 357.

[35] Cai, S.; Böck, D.; Pilhofer, M.; Gan, L. The in situ structures of mono-, di-, and trinucleosomes in human heterochromatin. Mol. Biol. Cell 2018, 29, 2450-2457. 
[36] Hsieh, T. H.; Weiner, A.; Lajoie, B.; Dekker, J.; Friedman, N.; Rando, O. J. Mapping Nucleosome Resolution Chromosome Folding in Yeast by Micro-C. Cell 2015, 162, 108-119.

[37] Grigoryev, S. A.; Bascom, G.; Buckwalter, J. M.; Schubert, M. B.; Woodcock, C. L.; Schlick, T. Hierarchical looping of zigzag nucleosome chains in metaphase chromosomes. Proc. Natl. Acad. Sci. U.S.A. 2016, 113, 1238-1243.

[38] Langmore, J. P.; Schutt, C. The higher order structure of chicken erythrocyte chromosomes in vivo. Nature 1980, 288, 620-622.

[39] Woodcock, C. L. Chromatin fibers observed in situ in frozen hydrated sections. Native fiber diameter is not correlated with nucleosome repeat length. J. Cell Biol. 1994, 125, 11-19.

[40] Kizilyaprak, C.; Spehner, D.; Devys, D.; Schultz, P. In vivo chromatin organization of mouse rod photoreceptors correlates with histone modifications. PLOS ONE 2010, 5, e11039.

[41] Scheffer, M. P.; Eltsov, M.; Bednar, J.; Frangakis, A. S. Nucleosomes stacked with aligned dyad axes are found in native compact chromatin in vitro. J. Struct. Biol. 2012, 178, 207-214.

[42] Zhou, K.; Gaullier, G.; Luger, K. Nucleosome structure and dynamics are coming of age. Nat. Struct. Mol. Biol. 2019, 26, 3-13.

[43] Chen, Y.; Tokuda, J. M.; Topping, T.; Meisburger, S. P.; Pabit, S. A.; Gloss, L. M.; Pollack, L. Asymmetric unwrapping of nucleosomal DNA propagates asymmetric opening and dissociation of the histone core. Proc. Natl. Acad. Sci. U.S.A. 2017, 114, 334-339.

[44] Parsons, T.; Zhang, B. Critical role of histone tail entropy in nucleosome unwinding. $J$ Chem Phys 2019, 150, 185103.

[45] Schlingman, D. J.; Mack, A. H.; Kamenetska, M.; Mochrie, S. G. J.; Regan, L. Routes to DNA accessibility: alternative pathways for nucleosome unwinding. Biophys. J. 2014, 107, 384-392.

[46] Alkhatib, S. G.; Landry, J. W. The nucleosome remodeling factor. FEBS Lett. 2011, 585, 3197-3207.

[47] Pentakota, S.; Zhou, K.; Smith, C.; Maffini, S.; Petrovic, A.; Morgan, G. P.; Weir, J. R.; Vetter, I. R.; Musacchio, A.; Luger, K. Decoding the centromeric nucleosome through CENP-N. Elife 2017, 6.

[48] Fyodorov, D. V.; Zhou, B. R.; Skoultchi, A. I.; Bai, Y. Emerging roles of linker histones in regulating chromatin structure and function. Nat. Rev. Mol. Cell Biol. 2018, 19, 192-206. 
[49] Talbert, P. B.; Henikoff, S. Histone variants on the move: substrates for chromatin dynamics. Nat. Rev. Mol. Cell Biol. 2017, 18, 115-126.

[50] Collepardo-Guevara, R.; Schlick, T. Chromatin fiber polymorphism triggered by variations of DNA linker lengths. Proc. Natl. Acad. Sci. U.S.A. 2014, 111, 8061-8066.

[51] Garcia-Saez, I.; Menoni, H.; Boopathi, R.; Shukla, M. S.; Soueidan, L.; Noirclerc-Savoye, M.; Le Roy, A.; Skoufias, D. A.; Bednar, J.; Hamiche, A. et al. Structure of an H1-Bound 6-Nucleosome Array Reveals an Untwisted Two-Start Chromatin Fiber Conformation. Mol. Cell 2018, 72, 902-915.

[52] Grigoryev, S. A.; Schubert, M. Unraveling the multiplex folding of nucleosome chains in higher order chromatin. Essays Biochem. 2019, 63, 109-121.

[53] Wong, H.; Victor, J. M.; Mozziconacci, J. An all-atom model of the chromatin fiber containing linker histones reveals a versatile structure tuned by the nucleosomal repeat length. PLoS ONE 2007, 2, e877.

[54] Ozer, G.; Luque, A.; Schlick, T. The chromatin fiber: multiscale problems and approaches. Curr. Opin. Struct. Biol. 2015, 31, 124-139.

[55] Maresca, T. J.; Freedman, B. S.; Heald, R. Histone H1 is essential for mitotic chromosome architecture and segregation in Xenopus laevis egg extracts. J. Cell Biol. 2005, 169, 859-869.

[56] Routh, A.; Sandin, S.; Rhodes, D. Nucleosome repeat length and linker histone stoichiometry determine chromatin fiber structure. Proc. Natl. Acad. Sci. U.S.A. 2008, 105, 8872-8877.

[57] Hergeth, S. P.; Schneider, R. The H1 linker histones: multifunctional proteins beyond the nucleosomal core particle. EMBO Rep. 2015, 16, 1439-1453.

[58] Fan, Y.; Nikitina, T.; Zhao, J.; Fleury, T. J.; Bhattacharyya, R.; Bouhassira, E. E.; Stein, A.; Woodcock, C. L.; Skoultchi, A. I. Histone H1 depletion in mammals alters global chromatin structure but causes specific changes in gene regulation. Cell 2005, 123, 1199-1212.

[59] Shen, X.; Gorovsky, M. A. Linker histone H1 regulates specific gene expression but not global transcription in vivo. Cell 1996, 86, 475-483.

[60] Lu, X.; Wontakal, S. N.; Kavi, H.; Kim, B. J.; Guzzardo, P. M.; Emelyanov, A. V.; Xu, N.; Hannon, G. J.; Zavadil, J.; Fyodorov, D. V. et al. Drosophila H1 regulates the genetic activity of heterochromatin by recruitment of $\mathrm{Su}(\mathrm{var}) 3-9$. Science 2013, 340, 78-81. 
[61] Lee, H.; Habas, R.; Abate-Shen, C. MSX1 cooperates with histone H1b for inhibition of transcription and myogenesis. Science 2004, 304, 1675-1678.

[62] Zhang, Y.; Khan, D.; Delling, J.; Tobiasch, E. Mechanisms underlying the osteo- and adipodifferentiation of human mesenchymal stem cells. Scientific WorldJournal 2012, 2012, 793823.

[63] Christophorou, M. A.; Castelo-Branco, G.; Halley-Stott, R. P.; Oliveira, C. S.; Loos, R.; Radzisheuskaya, A.; Mowen, K. A.; Bertone, P.; Silva, J. C.; Zernicka-Goetz, M. et al. Citrullination regulates pluripotency and histone H1 binding to chromatin. Nature 2014, 507, 104-108.

[64] Thorslund, T.; Ripplinger, A.; Hoffmann, S.; Wild, T.; Uckelmann, M.; Villumsen, B.; Narita, T.; Sixma, T. K.; Choudhary, C.; Bekker-Jensen, S. et al. Histone H1 couples initiation and amplification of ubiquitin signalling after DNA damage. Nature 2015, 527, 389-393.

[65] Konishi, A.; Shimizu, S.; Hirota, J.; Takao, T.; Fan, Y.; Matsuoka, Y.; Zhang, L.; Yoneda, Y.; Fujii, Y.; Skoultchi, A. I. et al. Involvement of histone H1.2 in apoptosis induced by DNA double-strand breaks. Cell 2003, 114, 673-688.

[66] McGhee, J. D.; Felsenfeld, G. Nucleosome structure. Annu. Rev. Biochem. 1980, 49, 1115-1156.

[67] Lever, M. A.; Th'ng, J. P.; Sun, X.; Hendzel, M. J. Rapid exchange of histone H1.1 on chromatin in living human cells. Nature 2000, 408, 873-876.

[68] Misteli, T.; Gunjan, A.; Hock, R.; Bustin, M.; Brown, D. T. Dynamic binding of histone H1 to chromatin in living cells. Nature 2000, 408, 877-881.

[69] Catez, F.; Ueda, T.; Bustin, M. Determinants of histone H1 mobility and chromatin binding in living cells. Nat. Struct. Mol. Biol. 2006, 13, 305-310.

[70] Shimamura, A.; Sapp, M.; Rodriguez-Campos, A.; Worcel, A. Histone H1 represses transcription from minichromosomes assembled in vitro. Mol. Cell. Biol. 1989, 9, 5573-5584.

[71] Laybourn, P. J.; Kadonaga, J. T. Role of nucleosomal cores and histone H1 in regulation of transcription by RNA polymerase II. Science 1991, 254, 238-245.

[72] O’Neill, T. E.; Meersseman, G.; Pennings, S.; Bradbury, E. M. Deposition of histone H1 onto reconstituted nucleosome arrays inhibits both initiation and elongation of transcripts by T7 RNA polymerase. Nucleic Acids Res. 1995, 23, 1075-1082. 
[73] Kale, S.; Goncearenco, A.; Markov, Y.; Landsman, D.; Panchenko, A. R. Molecular recognition of nucleosomes by binding partners. Curr. Opin. Struct. Biol. 2019, 56, 164-170.

[74] Mishra, L. N.; Hayes, J. J. A nucleosome-free region locally abrogates histone H1-dependent restriction of linker DNA accessibility in chromatin. J. Biol. Chem. 2018, 293, 19191-19200.

[75] Woods, D. C.; Wereszczynski, J. Elucidating the influence of linker histone variants on chromatosome dynamics and energetics. Nucleic Acids Res. 2020, 48, 3591-3604.

[76] Lu, X. J.; El Hassan, M. A.; Hunter, C. A. Structure and conformation of helical nucleic acids: analysis program (SCHNAaP). J. Mol. Biol. 1997, 273, 668-680.

[77] Olson, W. K.; Bansal, M.; Burley, S. K.; Dickerson, R. E.; Gerstein, M.; Harvey, S. C.; Heinemann, U.; Lu, X. J.; Neidle, S.; Shakked, Z. et al. A standard reference frame for the description of nucleic acid base-pair geometry. J. Mol. Biol. 2001, 313, 229-237.

[78] Lu, X. J.; Olson, W. K. 3DNA: a software package for the analysis, rebuilding and visualization of three-dimensional nucleic acid structures. Nucleic Acids Res. 2003, 31, 5108-5121.

[79] Davey, C. A.; Sargent, D. F.; Luger, K.; Maeder, A. W.; Richmond, T. J. Solvent mediated interactions in the structure of the nucleosome core particle at 1.9 a resolution. J. Mol. Biol. 2002, 319, 1097-1113.

[80] Shaytan, A. K.; Armeev, G. A.; Goncearenco, A.; Zhurkin, V. B.; Landsman, D.; Panchenko, A. R. Coupling between Histone Conformations and DNA Geometry in Nucleosomes on a Microsecond Timescale: Atomistic Insights into Nucleosome Functions. J. Mol. Biol. 2016, 428, 221-237.

[81] Lionnet, T.; Joubaud, S.; Lavery, R.; Bensimon, D.; Croquette, V. Wringing Out DNA. Phys. Rev. Lett. 2006, 96, 178102-1-178102-4.

[82] Gore, J.; Bryant, Z.; Nöllmann, M.; Le, M. U.; Cozzarelli, N. R.; Bustamante, C. DNA overwinds when stretched. Nature 2006, 442, 836-839.

[83] Lange, O. F.; Grubmüller, H. Generalized correlation for biomolecular dynamics. Proteins 2006, 62, 1053-1061.

[84] Wisniewski, J. R.; Zougman, A.; Krüger, S.; Mann, M. Mass spectrometric mapping of linker histone H1 variants reveals multiple acetylations, methylations, and phosphorylation as well as differences between cell culture and tissue. Mol. Cell Proteomics 2007, 6, 72-87. 
[85] Zhou, B. R.; Feng, H.; Ghirlando, R.; Li, S.; Schwieters, C. D.; Bai, Y. A Small Number of Residues Can Determine if Linker Histones Are Bound On or Off Dyad in the Chromatosome. J. Mol. Biol. 2016, 428, 3948-3959.

[86] Li, Y.; Li, Z.; Dong, L.; Tang, M.; Zhang, P.; Zhang, C.; Cao, Z.; Zhu, Q.; Chen, Y.; Wang, H. et al. Histone $\mathrm{H} 1$ acetylation at lysine 85 regulates chromatin condensation and genome stability upon DNA damage. Nucleic Acids Res. 2018, 46, 7716-7730.

[87] Öztürk, M. A.; Cojocaru, V.; Wade, R. C. Toward an Ensemble View of Chromatosome Structure: A Paradigm Shift from One to Many. Structure 2018, 26, 1050-1057.

[88] Stützer, A.; Liokatis, S.; Kiesel, A.; Schwarzer, D.; Sprangers, R.; Söding, J.; Selenko, P.; Fischle, W. Modulations of DNA Contacts by Linker Histones and Post-translational Modifications Determine the Mobility and Modifiability of Nucleosomal H3 Tails.

[89] Perišić, O.; Portillo-Ledesma, S.; Schlick, T. Sensitive effect of linker histone binding mode and subtype on chromatin condensation. Nucleic Acids Res. 2019,

[90] Bednar, J.; Garcia-Saez, I.; Boopathi, R.; Cutter, A. R.; Papai, G.; Reymer, A.; Syed, S. H.; Lone, I. N.; Tonchev, O.; Crucifix, C. et al. Structure and Dynamics of a 197 bp Nucleosome in Complex with Linker Histone H1. Mol. Cell 2017, 66, 384-397.

[91] Li, G.; Levitus, M.; Bustamante, C.; J., W. Rapid spontaneous accessibility of nucleosomal DNA. Nat. Struct. Mol. Biol. 2005, 12, 46-53.

[92] Tims, H.; Gurunathan, K.; Levitus, M.; J., W. Dynamics of Nucleosome Invasion by DNA Binding Proteins. J. Mol. Biol. 2011, 411, 430-438.

[93] North, J.; Shimko, J.; Javaid, S.; Mooney, A.; Shoffner, M.; Rose, S.; Bundschuh, R.; Fishel, R.; Ottesen, J.; Poirier, M. Regulation of the nucleosome unwrapping rate controls DNA accessibility. Nucleic Acids Res. 2012, 40, 10215-10227.

[94] Sali, A.; Blundell, T. L. Comparative protein modelling by satisfaction of spatial restraints. J. Mol. Biol. 1993, 234, 779-815.

[95] Pettersen, E. F.; Goddard, T. D.; Huang, C. C.; Couch, G. S.; Greenblatt, D. M.; Meng, E. C.; Ferrin, T. E. UCSF Chimera-a visualization system for exploratory research and analysis. $J$ Comput Chem 2004, 25, 1605-1612. 
[96] Case, D. A.; Betz, R. M.; Cerutti, D. S.; Cheatham, T. E.; Darden, T. A.; Duke, R. E.; Giese, T. J.; Gohlke, H.; Goetz, A. W.; Homeyer, N. et al. AMBER 2018; University of California, San Francisco, 2018.

[97] Wriggers, W. Conventions and workflows for using Situs. Acta Crystallogr. D Biol. Crystallogr. 2012, $68,344-351$.

[98] Wriggers, W.; Milligan, R. A.; McCammon, J. A. Situs: A package for docking crystal structures into low-resolution maps from electron microscopy. J. Struct. Biol. 1999, 125, 185-195.

[99] Lopez-Blanco, J. R.; Garzon, J. I.; Chacon, P. iMod: multipurpose normal mode analysis in internal coordinates. Bioinformatics 2011, 27, 2843-2850.

[100] Abraham, M.; Murtola, T.; Schulz, R.; Páll, S.; Smith, J. C.; Hess, B.; Lindahl, E. GROMACS: high performance molecular simulations through multi-level parallelism from laptops to supercomputers. SoftwareX 2015, 1-2, 19-25.

[101] Jorgensen, W. L.; Chandrasekhar, J.; Madura, J. D.; Impey, R. W.; Klein, M. L. Comparison of simple potential functions for simulating liquid water. J Chem Phys 1983, 79, 926-935.

[102] Mahoney, M. W.; Jorgensen, W. L. A five-site model for liquid water and the reproduction of the density anomaly by rigid, nonpolarizable potential functions. J Chem Phys 1983, 112, 8910-8922.

[103] Joung, I. S.; Cheatham, T. E. Determination of alkali and halide monovalent ion parameters for use in explicitly solvated biomolecular simulations. J Phys Chem B 2008, 112, 9020-9041.

[104] Joung, I. S.; Cheatham, T. E. Molecular dynamics simulations of the dynamic and energetic properties of alkali and halide ions using water-model-specific ion parameters. J Phys Chem B 2009, 113, 1327913290.

[105] Maier, J. A.; Martinez, C.; Kasavajhala, K.; Wickstrom, L.; Hauser, K. E.; Simmerling, C. ff14SB: Improving the Accuracy of Protein Side Chain and Backbone Parameters from ff99SB. J Chem Theory Comput 2015, 11, 3696-3713.

[106] Ivani, I.; Dans, P. D.; Noy, A.; Perez, A.; Faustino, I.; Hospital, A.; Walther, J.; Andrio, P.; Goni, R.; Balaceanu, A. et al. Parmbsc1: a refined force field for DNA simulations. Nat. Methods 2016, 13, $55-58$. 
[107] Darden, T.; York, D.; Pedersen, L. Particle mesh Ewald: An N·log(N) method for Ewald sums in large systems. J. Chem. Phys. 1993, 98, 10089-10092.

[108] Parrinello, M.; Rahman, A. Polymorphic transitions in single crystals: A new molecular dynamics method. J. Appl. Phys. 1981, 52-12.

[109] Towns, J.; Cockerill, T.; Dahan, M.; Foster, I.; Gaither, K.; Grimshaw, A.; Hazlewood, V.; Lathrop, S.; Lifka, D.; Peterson, G. et al. XSEDE: accelerating scientific discovery. Computing in Science E Engineering 2014, 16, 62-74.

[110] Dršata, T.; Pérez, A.; Orozco, M.; Morozov, A. V.; Šponer, J.; Lankaš, F. Structure, Stiffness and Substates of the Dickerson-Drew Dodecamer. J Chem Theory Comput 2013, 9, 707-721.

[111] Kraskov, A.; Stögbauer, H.; Grassberger, P. Estimating mutual information. Phys Rev E Stat Nonlin Soft Matter Phys 2004, 69, 066138.

[112] McClendon, C. L.; Hua, L.; Barreiro, A.; Jacobson, M. P. Comparing Conformational Ensembles Using the Kullback-Leibler Divergence Expansion. J Chem Theory Comput 2012, 8, 2115-2126.

[113] Roa, C. R.; Nayak, T. K. Cross Entropy, Dissimilarity Measure, and Characterizations of Quadratic Entropy. IEEE Trans. Inf. Theory 1985, 31, 589-593.

[114] Lin, J. Divergence Measures Based on the Shannon Entropy. IEEE Trans. Inf. Theory 1991, 37, $145-149$.

[115] Endres, D. M.; Schindelin, J. E. A new metric for probability distributions. IEEE Trans. Inf. Theory 2003, 49, 1858-1860.

[116] Österreicher, F.; Vajda, I. A new class of metric divergences on probability spaces and its applicability in statistics. Ann. Inst. Stat. Math 2003, 55, 639-653.

[117] Fuglede, B.; Topsoe, F. Jensen-Shannon divergence and Hilbert space embedding. International Symposium on Information Theory, 2004. ISIT 2004. Proceedings. 2004; p 31. 